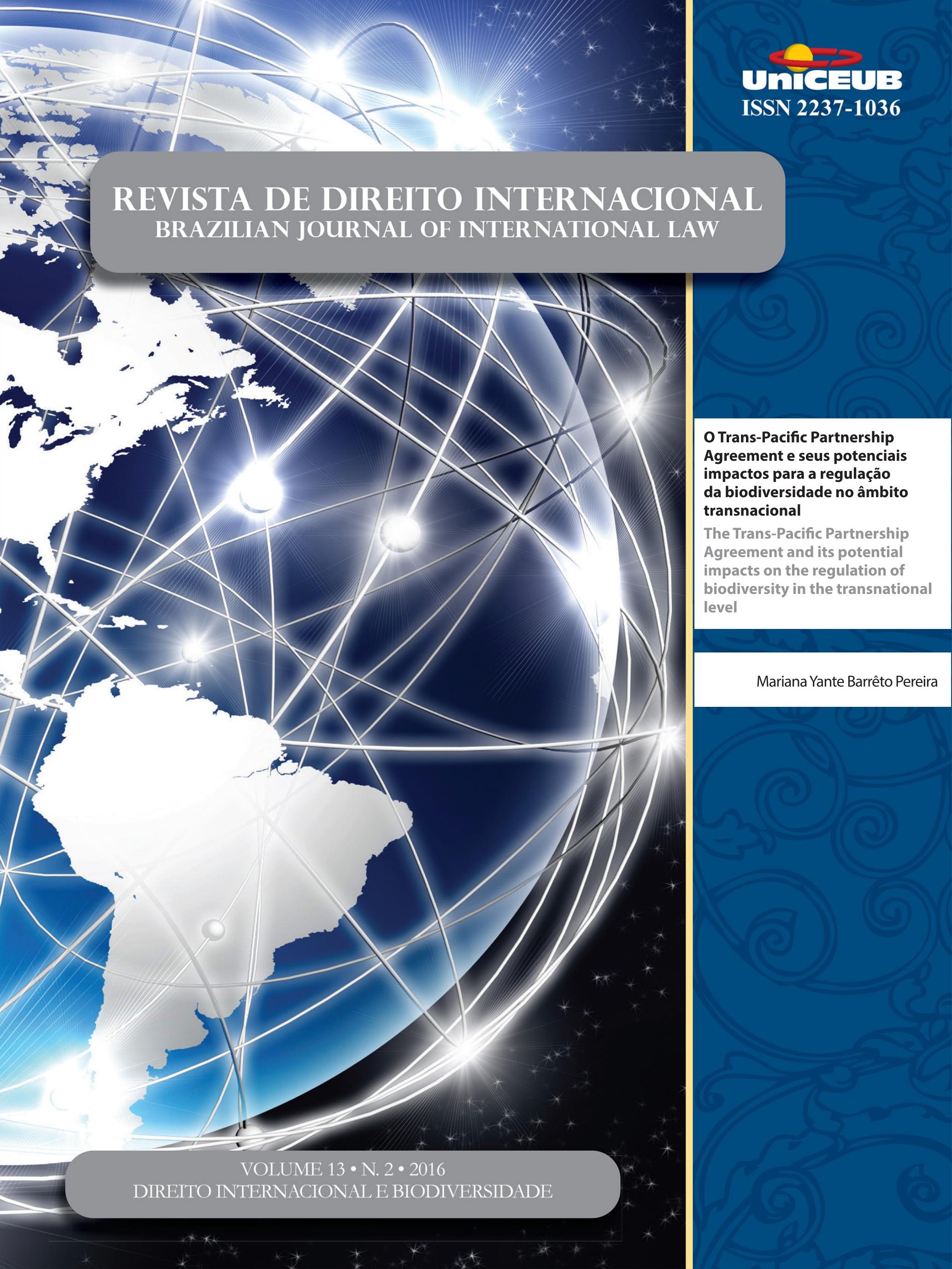




\section{Sumário}

CRÔNICAS DE DIREITO INTERNACIONAL PRIVADO 3 Gustavo Ferreira Ribeiro, Inez Lopes Matos Carneiro de Farias, Nadia de Araujo e Marcelo De Nardi

EDITORIAL 22

Márcia Dieguez Leuzinger e Solange Teles da Silva

IMPROVING THE EFFECTIVENESS OF LEGAL ARRANGEMENTS TO PROTECT BIODIVERSITY: AUSTRALIA AND BRAZIL .25

Paul Martin, Márcia Dieguez Leuzinger e Solange Teles da Silva

O RECONHECIMENTO DA DIGNIDADE DOS ELEMENTOS DA BIODIVERSIDADE COM BASE NO DIÁLOGO ENTRE O DIREITO INTERNACIONAL E O ORDENAMENTO JURÍDICO BRASILEIRO

Augusto César Leite de Resende

O REGIME INTERNACIONAL DO CLIMA E A PROTEÇÃO AOS “REFUgIADOS CLIMÁTICOS”: QUAIS DESAFIOS DA COP 21?

Ana Carolina Barbosa Pereira Matos e Tarin Cristino Frota Mont'Alverne

A anÁlise do MECANismo REdD+ COM Vistas À MitigaÇÃo dos EFEITOS DAS MUdANÇAS CLIMÁTICAS E À PROTEÇÃO DA DIVERSIDADE BIOLÓGICA FLORESTAL

Diogo Andreola Serraglio e Heline Sivini Ferreira

ECOLABELS DE EFICIÊNCIA ENERGÉtiCA E SUA CONSISTÊNCIA COM A DISCIPLINA DOS PPM's E PROVISÕES dOs Acordos GATT E TBT.

Cristiane Derani e Arthur Rodrigues Dalmarco

ANÁLISIS DEL ORDENAMIENTO JURÍDICO INTERNACIONAL SOBRE PROTECCIÓN DE LOS RECURSOS GENÉTICOS: DESAFIOS Y PERSPECTIVAS EN URUGUAY A PARTIR DE LA IMPLEMENTACIÓN DEL PROTOCOLO DE NAgOYA 115

Alina Celi 
LE RÉGIME INTERNATIONAL DE L'ACCÈS AUX RESSOURCES GÉNÉTIQUES AU PRISME DE L'ENTRÉE

en VIgUeUr du Protocole de NAgoya .............................................................. 131

Rodolpho Zahluth Bastos, Otávio Canto, Karine Galy e Isabelle Vestris

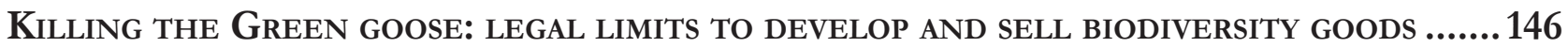
José Augusto Fontoura Costa e Liziane Paixão Silva Oliveira

VÍNCULO SUBSTANCIAL E AS BANDEIRAS DE CONVENIÊNCIA: CONSEQUÊNCIAS AMBIENTAIS DECORRENTES DOS NAVIOS COM REGISTROS ABERTOS ....................................................... 160

Marcos Edmar Ramos Alvares da Silva e André de Paiva Toledo

ANÁLISE DE CONTRATOS PÚblicos SOCIOAMBIENTAIS NO CENÁRIO PORTUGUÊS DE CRISE ECONÔ-

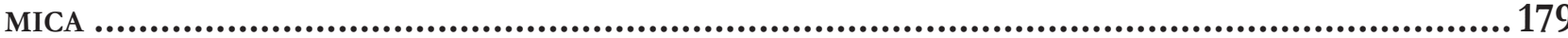

Alice Rocha da Silva e Matheus Passos Silva

A ORganizaÇão do TRATAdo DE COOPERAÇão AMAZÔNICA: UMA ANÁLISE CRÍTICA dAS RAZÕES POR TRÁS DA SUA CRIAÇÃO E EVOLUÇÃO

Paulo Henrique Faria Nunes

O uso de EXPERTS EM Controvérsias ambientais Perante a CorTe InTERnacional de JusTIÇA ........................................................................................................245

Lucas Carlos Lima

Os VIESES dA BIODIVERSIDAdE APRESENTAdos PELO CASO do PARQUE EÓlico DE BALd HiLls ......261 Natália Zampieri e Mariana Cabral

Mining CBD 275

Claire Lajaunie e Pierre Mazzega

Biotecnologia moderna, direito e o pensamento Abissal

Reichardt, F.V., Garavello, M. E. P. E., Molina, S.M.G. e Ballester, M. V. R.

Community Core Values como parâmetro de efetivação dos Princípios da Precaução e da Participação Popular em instrumentos de controle de projeto atividade de alta COMPLEXIDADE AMBIENTAL................................................................................... 314

Michelle Lucas Cardoso Balbino 
Discussões BIOTECNOLÓgICAS QUANTO AOS ORGANISMOS GENETICAMENTE MODIFICADOS NO ÂMBito da OMC: do contencioso ao acordo de vontades entre Argentina e União EuroPEIA

Gustavo Paschoal Oliveira

O Trans-Pacific Partnership Agreement e seus potenciais impactos para a regulação

DA BIODIVERSIDADE NO ÂMBITO TRANSNACIONAL..........................................................375

Mariana Yante Barrêto Pereira

AS ÁREAS PROTEGIDAS TRANSFRONTEIRIÇAS: REFLEXÕES CRÍTICAS ACERCA DE UM USO GEOPOLÍTICO DO DIREITO DA BIODIVERSIDADE.

Rabah Belaidi

O Que o caso Estados Unidos vs. Texas nos dirá sobre o direito de IMigração nos EsTADOS UNIDOS? 409

Danielle Anne Pamplona

Clóvis Beviláqua e a justiça internacional: entre o sim E o Não a Rui Barbosa.... .422 Paulo Emílio Vauthier Borges de Macedo

Possibilidade de delegação de atribuição para a Celebração de Tratados pela RepúbliCa Federativa do Brasil: análise do artigo 84, VIII c/c Parágrafo único da ConstituiÇão FEDERAL

Luciano Monti Favaro e Héctor Valverde Santana

Dignity, ubuntu, HUMANiTY AND AUTONOMOUS WEAPON SYSTEMS (AWS) DEBATE: AN AfRiCAN PERSPECTIVE 460

Thompson Chengeta

DiREITO INTERNACIONAL PRIVADO E O DIREITO TRANSNACIONAL: ENTRE A UNIFICAÇÃO E A ANARQUIA .503

André De Carvalho Ramos

A ilusória ausênCia do termo DépeÇage na JURisprudênCia brasileira de contratos INTERNACIONAIS

Gustavo Ferreira Ribeiro 
O EFEITO DIRETO DAS DIRETIVAS E OS DIREITOS FUNDAMENTAIS........................................535 Lucas Fonseca e Melo e José Levi Mello do Amaral Júnior

DA APLICABILIDADE DO BULK FACTORING AOS GRUPOS DE SOCIEDADES 565 Daniel Amin Ferraz e Leonardo Arêba Pinto

A Questão hermenêutica no direito das GENTES ................................................580 Inocêncio Mártires Coelho 


\title{
O Trans-Pacific Partnership Agreement e seus potenciais impactos para a regulação da biodiversidade no âmbito transnacional*
}

\author{
The Trans-Pacific Partnership Agreement and \\ its potential impacts on the regulation of \\ biodiversity in the transnational level
}

Mariana Yante Barrêto Pereira**

\section{Resumo}

O presente artigo visa analisar o regime de proteção ao meio ambiente, especificamente à biodiversidade, adotado no âmbito do Trans-Pacific Partnership agreement (TPP), considerando-se a inserção do tema no marco jurídico de um tratado de comércio e de investimentos e sua articulação com o sistema internacional de proteção ao meio ambiente. Pretende-se examinar, criticamente, os riscos advindos da transversalização de tais agendas, havendo sido priorizada a metodologia comparativa, tanto pelo caráter recente do acordo, assinado em fevereiro de 2016, quanto em relação aos impactos ocorridos, no âmbito de outros tratados bilaterais e regionais de escopo similar. $\mathrm{O}$ artigo estrutura-se em três eixos, a saber, a inserção da agenda ambiental no contexto de cooperação comercial e de proteção aos investimentos, no exame dos dispositivos do TPP quanto à biodiversidade em face do regime internacional existente e em observações sobre seu mecanismo de solução de controvérsias. Com base nesses elementos, são tecidas considerações sobre os impactos do TPP sobre a governança global em matéria ambiental, desde a retórica e da técnica legislativa utilizadas e de sua articulação com a emergente governança experimentalista.

Palavras-chave: TPP. Biodiversidade. Comércio. Investimento. Direito Ambiental.

* Recebido em 01/05/2016 Aprovado em 03/06/2016

** Doutoranda em Direito Internacional pela Wuhan University e em Relações Privadas Supranacionais pela Universidade Federal de Pernambuco. Pesquisadora do Instituto de Estudos da Ásia. Professora da Associação Caruaruense de Ensino Superior- ASCES/PE. E-mail: marianapereira@asces.edu.br.

\section{Abstract}

This article aims to analyze the system of protection of the environment, specifically the biodiversity, adopted within the framework of the TransPacific Partnership agreement (TPP), considering the inclusion of the issue in the legal framework of a trade and investment treaty and its articulation with the international system of protection of the environment. Its goal consists of examining critically the risks arising from the mainstreaming of such schedules, through the main adoption of a comparative methodology, due to the recent character of the agreement, signed in February 2016, as well as with regards to the impacts occurring in the framework of other bilateral and regional treaties of similar scope. The article is structured in 
three topics, namely, the insertion of the environmental agenda in the context of trade cooperation and investment protection, the examination of the provisions of the TPP regarding the biodiversity in the face of the existing international regime and on observations about your dispute resolution mechanism. From these elements, the impacts of the TPP on global environmental governance is briefly addressed, from the rhetoric and legislative technique used and their articulation with the emerging governance experimentalist.

Keywords: TPP. Biodiversity. Trade. Investment. Environmental Law.

\section{INTRODUÇão}

O Acordo de Associação Trans-Pacífico, mais conhecido pelo nome de Trans-Pacific Partnership agreement (TPP), consiste em um tratado internacional envolvendo doze países - Austrália, Brunei Darussalam, Canadá, Chile, Japão, Malásia, México, Nova Zelândia, Peru, Singapura, Estados Unidos da América e Vietnã.

Com a agregação, no âmbito de um mesmo acordo, de economias maduras, como Japão (último país a integrar as negociações, em 2013), Estados Unidos e Canadá, e de atores dominantes ou emergentes da Oceania, América Latina e a inclusão de Tigres Asiáticos (Singapura e Malásia), estima-se que o tratado de livre-comércio possa reconfigurar a dinâmica dos processos de cooperação regional e multilateral.

Ao mesmo tempo em que incorpora agendas atinentes à unificação de normas trabalhistas e à elevação de standards protetivos à propriedade intelectual e aos investimentos, o potencial do TPP em criar um arcabouço jurídico e institucional para as questões que abrange traduz-se na sua pungência econômica: 40\% da economia mundial, representando um terço das exportações e com um mercado consumidor de aproximadamente 800 milhões de indivíduos os quais, até 2025, devem movimentar 223 bilhões de dólares/ano.

Com o texto final concluído em Atlanta, Estados Unidos, e publicado em novembro de 2015, o acordo foi assinado pelos Estados negociantes, no último mês de fevereiro, na Nova Zelândia, que é sua formal depositária.

O TPP entrará em vigor internacional dentro de dois anos após todos os signatários notificarem que concluíram as reestruturações em seu ordenamento jurídico, ou, ainda, após dois anos e três meses se, ao menos seis deles, incluindo os Estados Unidos e o Japão, o tiverem efetuado.

Com trinta capítulos e diversos anexos, o tratado demanda reformas substanciais nas legislações domésticas, abrangendo mesmo o nível constitucional, na medida em que se buscou regulamentar diversas questões que pudessem estar direta ou indiretamente atreladas ao seu escopo de promoção do comércio e da proteção dos investimentos realizados interpartes.

Em relação a essa agenda transversal, questões eminentemente relacionadas aos Direitos Sociais (especificamente trabalhistas) e ao Direito Ambiental foram, também, objeto de capítulos próprios, sob o argumento por parte dos governos de que sua presença no acordo recrudesceria os compromissos estabelecidos nesses campos.

O discurso político, entretanto, discrepa das críticas que sua análise vem levantando, tanto no âmbito da sociedade civil como no espaço da academia, que apontam o instrumento como um possível retrocesso aos avanços normativos já conquistados na seara internacional.

O objetivo do presente artigo é analisar o texto do tratado e os criticismos estabelecidos desde a regulamentação da temática da governança em matéria de meio ambiente, notadamente sobre a biodiversidade, considerando-se os aspectos jurídicos que lhe são inerentes.

Uma das críticas que vêm sendo reiteradas com mais força entre a comunidade ambientalista é o caráter retórico do texto, ante a opção por expressões recomendatórias e a ausência de mecanismos de uniformização e recrudescimento da agenda de proteção ambiental dos países signatários.

É importante observar, contudo, que tais escolhas terminológicas não pautaram a integralidade do texto; outros capítulos, como os comentados dispositivos sobre propriedade intelectual, notabilizaram-se, justamente, por seu caráter mandatório e por ampliar, substantivamente, os direitos já reconhecidos pelos membros com base nas legislações domésticas.

Nesse sentido, quando o esboço do capítulo destinado ao meio ambiente foi antecipadamente revelado pelo Wikileaks $^{1}$ - alguns meses depois de o texto provisório

1 O texto normativo utilizado é o disponível no sítio eletrônico do 
atinente à propriedade intelectual vir à tona - a plataforma publicou uma análise, da qual se destaca:

On 13 November last year, WikiLeaks released the secret draft text of the Intellectual Property Rights Chapter, which showed how nations were forced to change laws and to prosecute in defence (sic) of the biggest corporate interests in the field of IP rights.

In sharp contrast, the Environment Chapter does not include enforcement mechanisms serving the defence (sic) of the environment; it is vague and weak, and adheres to the lowest common denominator of environmental interests.

The word "appropriate" is found in various forms in 43 places in the draft text, in such contexts as: "Where possible and appropriate, the Parties shall seek to complement and utilise (sic) their existing cooperation mechanisms and take into account relevant work of regional and international organizations." The word "may" is also found 43 times in the 23-page draft. ${ }^{2}$

Como se discutirá adiante, porém, não é apenas a indefinição dos compromissos ambientais que pode ser discutida em termos jurídicos. Existem reflexões a serem tecidas com base na contextualização da agenda de proteção à biodiversidade no âmbito de outros deveres aos quais os Estados signatários se submeteram naquela seara.

Os Estados-partes do TPP são, parcialmente, ou em sua integralidade, também, signatários de outros acordos em matéria de proteção ao meio ambiente, seja pela tutela direta à biodiversidade, seja pela redução de fatores que a comprometem, como a poluição marinha e do ar.

Pretende-se discutir, dessa forma, em que medida a redação do TPP contribui para ratificar ou mesmo recrudescer os deveres dos países no que tange a essas questões, conforme o fez com os objetivos atinentes à propriedade intelectual, por exemplo.

United States Trade Representative: $<$ https://ustr.gov/tpp/>.

2 Livre tradução do original: Em 13 de novembro do ano passado, o WikiLeaks revelou o esboço secreto do capítulo relativo aos Direitos de Propriedade Intelectual, o qual demonstrou como as nações seriam forçadas a modificar legislações e perseguir em defesa dos grandes interesses das corporações em matéria de Propriedade Intelectual. Em um contraste marcante, o capítulo sobre o Meio Ambiente não inclui mecanismos de implementação que funcionem em defesa do meio ambiente; é vago e fraco, e adere ao mínimo denominador comum quanto aos interesses ambientais. A palavra "apropriado" é encontrada sob diversas formas em 43 lugares do esboço, em contextos como: 'quando possível e apropriado, as Partes devem procurar complementar e utilizar seus mecanismos de cooperação existentes e levar em consideração o trabalho relevante das organizações internacionais e regionais'. O modal 'poder' é, também, encontrado 43 vezes no esboço de 23 páginas.
O enfoque a ser adotado é a análise dessas interfaces desde a tendência dos Acordos Bilaterais, Regionais e Transnacionais de Comércio e de Investimento de buscarem regulamentar aspectos relativos a outros ramos do Direito e do Direito Internacional, incluindo a utilização de um mecanismo único de solução de controvérsias para o exame conjunto dessas questões.

\section{A Concatenação das Agendas ECONÔMica E AMBIENTAL: AVANÇO OU RETROCESSO?}

Em termos estruturais, o TPP como acordo transnacional de comércio e de investimentos parece seguir uma tendência de se abarcar sob a ideia de um tratado 'guarda-chuva' agendas que, de forma transversal, podem influenciar na concretização dos objetivos econômicos de cooperação e liberalização.

Conforme ocorrera nas tratativas em torno da Rodada Uruguai - a qual resultou na assinatura do Acordo de Marrakesh, que instituiu a Organização Mundial do Comércio - as discussões para englobar no tratado a pauta atinente à propriedade intelectual partiram da concepção de que regras domésticas para sua proteção poderiam servir como barreiras não tarifárias.

Da mesma maneira, desde 1995, com a entrada em vigor do tratado e o início dos trabalhos do Órgão de Solução de Controvérsias, cada vez mais, questões atinentes ao Direito Ambiental (notadamente à proteção à biodiversidade) e aos Direitos Humanos vêm sendo invocadas pelas partes litigantes e, consequentemente, enfrentadas na resolução dos casos em disputa.

É importante observar que, em relação ao nível do direito material, muitas questões não foram incorporadas aos instrumentos jurídicos da OMC em virtude de não terem sido alcançados níveis de consenso mínimo, mas subsiste a possibilidade de que sejam enfrentadas pelo Órgão de Solução de Controvérsias diante de eventual enquadramento das medidas refutadas pelos Estados partes no Artigo XXIV do GATT, sobretudo. Ainda assim, é necessário que os aspectos ventilados se insiram no âmbito do comércio de bens e serviços, o que exclui algumas questões de fundo importantes, como investimentos.

Por outro lado, e, também, devido às limitações no alcance temático das tratativas da OMC, a assimilação 
de temáticas atreladas ao manejo dos recursos naturais e à proteção da biodiversidade pelos compromissos de cooperação em matéria comercial não se dá, apenas, em nível multilateral.

O Acordo Regional de Comércio (ARC) entre Colômbia e Estados Unidos ${ }^{3}$, por exemplo, apresenta diversos dispositivos que provocam impactos domésticos, sobretudo em relação à Colômbia, quanto à utilização de modelos sustentáveis de produtos agrícolas, por exemplo. Com a abertura dos mercados para os produtos estadunidenses, a agricultura orgânico-familiar e os(as) pequenos(as) produtores(as), próprios(as) de muitas regiões rurais daquele país, perdeu espaço, sem incentivos para a produção, que terminaria violando o princípio do tratamento nacional ${ }^{4}$.

No que tange aos Tratados Bilaterais de Investimento (mais conhecidos por sua sigla em inglês - BITs), uma breve análise dos casos sob a jurisdição do mecanismo de solução de controvérsias do Grupo Banco Mundial, a saber, o International Centre for Settlement of Investment Disputes (ICSID), demonstra que são, igualmente, rechaçadas tentativas de um dos Estados pactuantes utilizarem a proteção de seus recursos naturais para balizar eventual negativa de cumprimento de suas obrigações.

$\mathrm{Na}$ medida em que a exploração de tais recursos também é abarcada pelo conceito de investimento, emergem dispositivos no tratado que tendem a salvaguardar qualquer iniciativa do Estado-membro de retirá-los do âmbito de influência dos contratos porventura pactuados. À ilustração, em 2000, foi assinado o Acordo entre o Governo da República Federativa da Etiópia e o Governo da República do Sudão para a Promoção e Proteção Recíproca de Investimento ${ }^{5}$, que, em sua definição de investimento, incluiu "concessões sobre a procura por, cultivar, extrair ou explorar recursos

3 United States-Colombia Trade Promotion Agreement. Assinado em 22 de novembro de 2006, e aprovado pelo Congresso colombiano em 2007, com revisão e aprovação da Corte Constitucional deste país em julho de 2008. O acordo, entretanto, apenas entrou em vigor em 15 de maio de 2012, após a aprovação pelas casas legislativas estadunidenses.

4 FRIENDS FOR EARTH. The Colombia FTA: bad for the environment. Available in: <www.ffe.org>. Access in: 5 abr. 2016.

5 Agreement Between the Government of the Federal Democratic Republic of Ethiopia and the Government of the Republic of Sudan on the Reciprocal Promotion and Protection of Investment, Ethiopia and Sudan. naturais" "O acordo acrescenta que uma compensação "imediata, adequada e efetiva"7 pode ser devida se uma das nações realiza "quaisquer medidas de expropriação, nacionalização ou quaisquer outras medidas da mesma natureza ou com o mesmo efeito contra o investimento de Investidores da outra Parte Contratante" .

O já mencionado ARC entre Colômbia e Estados Unidos - que também contém um capítulo sobre investimentos - é apontado pelos (as) ambientalistas desde sua assinatura como uma séria ameaça à biodiversidade. $\mathrm{O}$ argumento central consiste em que o acordo encorajou a expansão massiva das plantations de óleo de palma, que, somente na região de Chocó - com 145 mil km² de biota distribuída em floresta de planície, tropical, montanhas e costa -, 154 mil hectares são destruídos anualmente por atividades da mesma natureza. ${ }^{9}$ No mesmo sentido, o estímulo às atividades de mineração e extração de petróleo vem sendo vinculado à destruição de ecossistemas e ao deslocamento forçado de comunidades locais; de acordo com o que foi reportado no sítio eletrônico das Nações Unidas ${ }^{10}$, ao menos mil membros das comunidades das regiões do Rio Anchicayá e de Chocó emigraram forçadamente devido à violência imposta pelos controladores das atividades mineradoras.

No caso do TPP, a análise das rodadas de negociação evidencia que a temática ambiental foi suscitada desde o primeiro encontro, realizado em março de 2010, na Austrália. Na ocasião, estabeleceu-se uma controvérsia quanto à utilização do Acordo de Cooperação Ambiental assinado no marco da Acordo de Parceria Econômica Estratégica Trans-Pacífico (mencionado nas tratativas como Pacific Four ou P4), assinado em 2005 por Brunei, Chile, Nova Zelândia e Singapura.

As divergências se deram, justamente, pela simplicidade institucional adotada pelo último, pois se assinalou

6 Livre tradução do original: 'concessions to search for, cultivate, extract or exploit natural resources'.

7 Livre tradução do original: 'prompt, adequate and effective compensation'.

8 Livre tradução do original: 'any measures of expropriation, nationalization or any other measures having the same nature or the same effect against investment of Investors of the other Contracting Party'.

9 GARCIA, Cristian. Colombia: the Palm Trees' Bloody Oil. Tropenwald, 2007. Available in: <http://www.robinwood.de/german. magazine/200703/94-3-7-kolum.eng.pdf.> . Access in: 20 abr. 2016. 10 UN News Center, Violent Struggle over Illegal Mining in Colombia Forces Hundreds to Flee, Available in: <http://www.un.org/ apps $/$ news $/$ story.asp?NewsID $=37796 \& C r=$ colombia\&Cr1 $=>$. Access in: 20 abr. 2016. 
que, devido às restrições orçamentárias, os projetos voltados para cooperação em matéria ambiental são financiados pelos Estados-membros que os integram. ${ }^{11}$

Um novo destaque à agenda ambiental deu-se a partir da terceira rodada de negociações, ainda em 2010, em Brunei Darussalam, para a qual foram convidados(as) especialistas, buscando-se articulá-la às questões de comércio, serviços e investimentos. ${ }^{12}$

Essa inserção reflete, na verdade, uma tendência de, no âmbito dos acordos megarregionais, ampliar a pauta de negociações em relação ao que está abrangido pela Organização Mundial do Comércio, agenda que ficou conhecida como "WTO+", como referência ao acrônimo da organização em inglês.

A extensão ocorre, essencialmente, de duas maneiras - elevando-se os standards existentes quanto ao regime jurídico já estabelecido ou acoplando novas temáticas a serem deliberadas.

À ilustração da primeira, tem-se o mencionado sistema de proteção à propriedade intelectual, o qual, embora objeto do anexo sobre Aspectos dos Direitos de Propriedade Intelectual Relacionados ao Comércio (Acordo TRIPS) e do sistema europeu tutelado pela Organização Mundial da Propriedade Intelectual (mais conhecida por sua sigla em inglês, WIPO), teve o recrudescimento de direitos conferidos aos detentores de patentes, por exemplo. É possível, ainda, ir além em questões cuja barganha - embora iniciada ou compreendida em sucessivas rodadas de negociação - encontra-se estagnada na seara multilateral por força da discrepância de alguns países envolvidos. É o caso dos subsídios à exportação de produtos agrícolas, geralmente levados à discussão por Austrália e Nova Zelândia (signatárias do TPP), que podem encontrar espaço de negociação fora do âmbito da OMC.

No que concerne ao segundo aspecto, o fato de os países-membros da OMC não haverem, quando da Rodada Uruguai, chegado a um lastro mínimo consensual em matéria de investimento fez com que seu regime

11 TRANS-PACIFIC PARTNERSHIP. Acuerdo de negociación transpacífico: informe de la primera ronda de negociación. Melbourne: Australia, 2010.

12 CHILE. Dirección de Relaciones Económicas Internacionales de Chile. Minuta de las negociaciones del TPP. Disponible en: < https:// www.direcon.gob.cl/wp-content/uploads/2014/05/MINUTA_ DE_LAS_NEGOCIACIONES_DEL_TPP.pdf>. Acceso en: 20 maio 2016 fosse deslocado para os acordos bilaterais e regionais e, mais atualmente, com a negociação de parcerias transoceânicas, também venha sendo incorporado nos tratados megarregionais, como o TPP e, ainda, em negociação Transatlantic Trade and Investment Partnership (TTIP), que, também, abarca a União Europeia.

Por seu turno, o impulso a tratativas voltadas para a criação de um arcabouço que garantisse segurança jurídica aos atores transnacionais privados levou à incorporação de um maior número de temas, sob a ótica de sua potencial influência na efetivação dos compromissos estabelecidos pelos Estados em matéria de comércio e de investimentos:

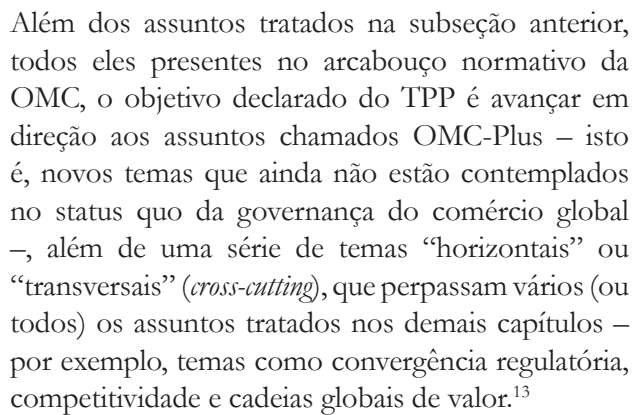

Em meio a esses aspectos, e a despeito das resistências apresentadas por países em desenvolvimento como Brunei e Vietnã, a pauta atinente aos direitos trabalhistas e ao meio ambiente foi inserida como decorrência, sobretudo, de uma pressão por parte dos Estados Unidos, cujo Poder Executivo, por força de um acordo bipartidário estipulado por seu Congresso desde 2007, deve seguir diretrizes na negociação de acordos de comércio ${ }^{14}$.

No que tange às questões ambientais e, notadamente, aos elementos relativos à biodiversidade, o próximo capítulo discutirá o aporte adotado pelos dispositivos do TPP.

\section{MeIo ambiente E BIODIVERSIDAde no TPP}

Sem prejuízo formal, evidentemente, dos demais Acordos Multilaterais em Matéria Ambiental dos quais

13 CARNEIRO, Flavio L. A Parceria transpacífica: principais características e impactos sobre a regulação do comércio mundial. Boletim de Economia e Política Internacional, n. 18, p. 60-72, set./dez. 2014. p. 66.

14 CARNEIRO, Flavio L. A Parceria transpacífica: principais características e impactos sobre a regulação do comércio mundial. Boletim de Economia e Política Internacional, n. 18, p. 60-72, set./dez. 2014. p. 67. 
os países-membros do TPP são signatários, o tratado previu um capítulo próprio destinado ao meio ambiente, que vêm provocando discussões e controvérsias no âmbito da comunidade ambientalista.

Todos os doze países signatários do TPP o são, também, da Convenção sobre o Comércio Internacional de Espécies da Fauna e Flora Selvagens em Perigo de Extinção (CITES) ${ }^{15}$, de 1975, que, atualmente, conta com 175 Estados-membros.

Provavelmente, o excerto mais cogente do texto em termos de compromisso ambiental corresponde ao dever de implementação das obrigações atinentes ao mencionado tratado, sumarizadas no Artigo 20.17 (Conservação e Comércio), do qual se destaca:

2. Accordingly, each Party shall adopt, maintain and implement laws, regulations and any other measures to fulfill its obligations under the Convention on International Trade in Endangered Species of Wild Fauna and Flora (CITES).

3. The Parties commit to promote conservation and to combat the illegal take of, and illegal trade in, wild fauna and flora.To that end, the Parties shall:

(a) exchange information and experiences on issues of mutual interest related to combating the illegal take of, and illegal trade in, wild fauna and flora, including combating illegal logging and associated illegal trade, and promoting the legal trade in associated products;

(b) undertake, as appropriate, joint activities on conservation issues of mutual interest, including through relevant regional and international fora; and

(c) endeavour to implement, as appropriate, CITES resolutions that aim to protect and conserve species whose survival is threatened by international trade.

O mesmo artigo ressalta, ainda, o compromisso de os Estados tomarem as medidas apropriadas para a proteção e conservação da fauna e flora identificadas como ameaçadas de extinção dentro de seu território [4.(a)] e manterem ou estabelecerem capacidades governamentais para o manejo sustentável de florestas e conservação da biodiversidade, além de envidar "esforços" (endeavour) para o fortalecimento da participação pública e da transparência no âmbito de tais mecanismos institucionais [4.(b)].

Interessante é observar, contudo, que existe um claro contraste presente no caput dos mencionados dispo-

15 Disponível em língua portuguesa em: < http://www.gddc.pt/ siii/docs/dec50-1980.pdf>. Acesso em: 25 abr. 2016. sitivos e as respectivas alíneas com a ideia de estabelecer obrigações mandatórias, na medida em que o texto ameniza a ideia de compromisso (commitment) com o uso da expressão "esforço" (endeavour), como o mencionado item 4.(b), e também a exemplo do que ocorre com o item 4.(c), que dispõe sobre o esforço para desenvolver e fortalecer a cooperação e mecanismos de consulta direcionados a entidades não governamentais interes$\operatorname{sadas}^{16}$.

Por outro lado, talvez a passagem que sumarize os anseios de que o regime estabelecido pelo TPP, na prática, colida com os deveres aos quais se submeteram os Estados partes na liberalização comercial e em matéria de investimentos seja o conceito de sustentabilidade adotado pelo tratado:

The TPPA measures intended to advance the environment are generally contained in chapter 20, titled Environment. The weak objectives for the chapter signal the poverty of serious disciplines to follow. Notably, the key concept of sustainable development is addressed there in the following terms: "the Parties recognize that enhanced cooperation to protect and conserve the environment and sustainably manage their natural resources brings benefits that can contribute to sustainable development" (emphasis added).3 Sustainable development cannot even be a clean objective in its own right. Implicitly, unsustainable development is permitted. ${ }^{17} 18$

16 4. Each Party further commits to: (a) take appropriate measures to protect and conserve wild fauna and flora that it has identified to be at risk within its territory, including measures to conserve the ecological integrity of specially protected natural areas, for example wetlands; (b) maintain or strengthen government capacity and institutional frameworks to promote sustainable forest management and wild fauna and flora conservation, and endeavour to enhance public participation and transparency in these institutional frameworks; and (c) endeavour to develop and strengthen cooperation and consultation with interested non-governmental entities in order to enhance implementation of measures to combat the illegal take of, and illegal trade in, wild fauna and flora.

17 Livre tradução do original: A maioria das provisões do TPP atinentes ao avanço das questões ambientais está prevista no capítulo 20, intitulado Meio Ambiente. Os objetivos fracos do capítulo assinalam a pobreza da disciplina normativa que os seguem. Notavelmente, o conceito-chave de desenvolvimento sustentável é abordado nos seguintes termos: "As partes reconhecem que a cooperação reforçada para proteger e conservar o meio ambiente e gerenciar sustentavelmente seus recursos naturais traz benefícios que podem contribuir para o desenvolvimento sustentável" (grifo nosso). $3 \mathrm{O}$ desenvolvimento sustentável não pode sequer ser um objetivo em si mesmo. Implicitamente, o desenvolvimento insustentável é permitido.

18 TERRY, Simon. The environment under TPPA governance: the law foundation New Zealand. Available in: <http://www.sustainabilitynz.org/wp-content/uploads/2016/01/TheEnvironmentUnderT- 
Essa crítica já era endereçada à Convenção das $\mathrm{Na}$ ções Unidas sobre Diversidade Biológica (CDB), na medida em que, ao mesmo tempo em que foi desenhada como uma legislação compreensiva diante de um até então fragmentado contexto normativo sobre proteção à diversidade, deixou de dar o enfoque protetivo que o tema deveria merecer.

De acordo com Guruswamy ${ }^{19}$, a aludida Convenção refutou o conceito de desenvolvimento sustentável ao enfatizar a concepção de crescimento econômico em detrimento da proteção ambiental, dando espaço para que recursos àquele destinados terminem por causar danos à biodiversidade.

Apesar das fragilidades apontadas e de, na prática, a CDB prescindir de suficientes dispositivos substantivos, o autor salienta que a Convenção teve inegável relevância no contexto dos compromissos ambientais até então estabelecidos - considerando, ainda, a Agenda 21 na qual sua negociação esteve inserida - por seu caráter cogente. Em contraponto a muitos instrumentos característicos do regime internacional de proteção do meio ambiente, o tratado estabeleceu um sistema vinculante (hard law) quanto às obrigações atinentes à biodiversidade.

A despeito de dez dos doze países que compõem o TPP tenham assumido obrigações internacionais de proteção à flora e à fauna no âmbito da Convenção sobre Diversidade Biológica, o caput do artigo menciona o compromisso de combater (combat) seu comércio ilegal, e não de proibi-lo.

Outra questão importante diz respeito à proteção da biodiversidade marinha. Se, por um lado, o TPP faz referência às obrigações estabelecidas no âmbito da CITES, por outro, essa convenção deixa em aberto a regulação das questões transversais ao Direito do Mar:

ART. XIV (6) - Nenhuma disposição da presente Convenção prejudica a codificação e elaboração do direito do mar pela Conferência das Nações Unidas sobre o Direito do Mar, convocada em virtude da resolução n. ${ }^{\circ} 2750 \mathrm{C}(\mathrm{XXV})$ da Assembleia Geral das Nações Unidas, nem as reivindicações e posições jurídicas, presentes ou futuras, de qualquer Estado no que respeita ao direito do mar, e a natureza e alcance da sua jurisdição costeira e da

PPAGovernance_2016.pdf>. Access: 20 abr. 2016.p. 6.

19 GURUSWAMY, Lakshman D. The convention on biological diversity: a polemic. In: GURUSWAMY, Lakshman D; MCNEELY, Jeffrey A. (Eds.). Protection of global biodiversity: converging strategies. London: Duke University Press, 1998. p. 351-359. jurisdição que ele exerce sobre os navios arvorando a sua bandeira.

Conquanto, evidentemente, a redação do mencionado dispositivo seja suficientemente abrangente - mesmo porque, à época de sua elaboração e entrada em vigor, sequer estavam definidos os termos da Convenção das Nações Unidas sobre o Direito do Mar (CNUDM), de 1982 - o TPP deu continuidade às controvérsias normativas atinentes ao manejo sustentável da biodiversidade marinha.

De fato, o uso sustentável dos recursos biológicos que se encontram no escopo territorial dos Estados é objeto da Convenção sobre Diversidade Biológica, a qual, embora assinada por 175 países, não foi ratificada, por exemplo, pelos Estados Unidos. Outro país que faz parte do TPP e que possui uma situação indefinida quanto à CDB é o Japão (apenas assinou o tratado, em 1993).

A implementação integral dos compromissos decorrentes do acordo enfrenta largos debates entre os países, sobretudo porque após as tratativas da Rodada Uruguai a assinatura do Tratado de Marrakesh, instaurou-se um regime colidente com o previamente pela CDB. Devido ao princípio do single-undertaking, quaisquer Estados que aderem à Organização Mundial do Comércio tornam-se, também, parte do Acordo sobre Aspectos dos Direitos de Propriedade Intelectual Relacionados ao Comércio (mais conhecido por sua sigla em inglês TRIPS - Agreement on Trade-Related Intellectual Property Rights).

Ocorre, porém, que, enquanto o CDB baliza-se nos princípios de repartição justa e equitativa dos benefícios decorrentes da exploração de recursos genéticos (Artigo 1), incluindo a valorização dos conhecimentos tradicionais, o TRIPS adota uma perspectiva oposta, focada na proteção dos direitos de propriedade intelectual de quem detenha ou desenvolva produtos e tecnologias, ainda que resultantes de conhecimento tradicional.

Ainda que a CDB se utilize, frequentemente, de uma terminologia pouco cogente, é inegável que houve avanços nas discussões em torno da efetivação do mencionado princípio, ilustrados pela assinatura do Protocolo de Nagoya sobre Acesso a Recursos Genéticos e a Repartição Justa e Equitativa dos Benefícios Advindos de sua Utilização à Convenção sobre Diversidade Biológica, em vigor internacional a partir de 12 de outubro de 2014. Conquanto a linguagem mantenha o tom predominantemente recomendatório já encontrado na $\mathrm{CDB}$, 
sua relevância política no âmbito das Relações Internacionais é inegável.

É importante lembrar, ainda, que Brunei Darussalam, Estados Unidos e Japão são membros originários da OMC (submetidos a seu regime desde 1 de janeiro de 1995), e, portanto, vinculados ao sistema normativo do TRIPS.

Embora os dispositivos relativos à proteção do patrimônio genético tenham aplicabilidade a quaisquer recursos da biota, no caso dos países signatários do TPP, a biodiversidade marinha é ora citada devido ao fato de determinados compromissos ambientais também colidirem com a sutileza normativa do acordo.

O exemplo mais ilustrativo é, certamente, a exclusão de qualquer menção à Convenção Internacional para a Regulação da Atividade Baleeira, destinada à regulação do comércio das baleias e à proteção da espécie. Após a proibição definitiva da caça das baleias em 1985, o país se utilizava de um argumento científico para conferir licenças que terminavam em permiti-la na prática. ${ }^{20} \mathrm{Em}$ 2014, porém, a Corte Internacional de Justiça, demandada pela Austrália e com intervenção da Nova Zelândia, apreciou o caso com base na análise do programa baleeiro instituído pelo Japão na região antártica, entendendo que era de natureza comercial e abusava de uma exceção científica estabelecida no âmbito da moratória internacional de pesca baleeira de 1986. Dessa forma, seria incompatível com as obrigações assumidas no âmbito da Convenção. ${ }^{21}$

Ao final do mesmo ano, porém, o país anunciou que daria continuidade à emissão das licenças, que seriam balizadas em um critério revisado, sob o argumento de que a interpretação dada à decisão da CIJ era distinta, estendendo, em mais um ano, a moratória que havia sido declarada, também sob a autoridade da International Whaling Comission. ${ }^{22}$ Em 2015, A Comissão pediu informações adicionais ao Japão acerca do novo programa,

20 INTERNATIONAL WHALING COMMISSION. Commercial Whaling. Available in: <https://iwc.int/commercial>. Access in: 13 abr. 2016.

21 INTERNATIONAL COURT OF JUSTICE. Whaling in the Antartic(Australiav:Japan:NewZealandintervening). Availablein: $<$ http:/ / www.icj-cij.org $/$ docket $/$ index.php?p1 $=3 \& p 2=1 \&$ case $=148>$. Access in: 13 abr. 2016.

22 NELSEN, Arthur. Japan Defies IWC Ruling on "Scientific Whaling". The Guardian. 2014. Available in: <http://www.theguardian.com/environment/2014/sep/18/japan-whaling-southernocean-iwc-ruling >. Access: 15 abr. 2016. na medida em que o Estado anunciara a retomada das atividades para o período de 2015-2016. ${ }^{23}$

Conquanto do mencionado Tratado façam também parte a Austrália, o Chile, o México, Nova Zelândia, Peru e Estados Unidos, o dispositivo do TPP dedicado à proteção da biodiversidade marinha optou por uma terminologia que deixou a seus membros o juízo discricionário em torno das medidas a serem tomadas, alijadas da natureza de outras obrigações porventura assumidas internacionalmente:

Art. 20.16.4 Each Party shall promote the long-term conservation of sharks, marine turtles, seabirds, and marine mammals, through the implementation and effective enforcement of conservation and management measures. Such measures should include, as appropriate:

(a) for sharks: the collection of species specific data, fisheries bycatch mitigation measures, catch limits, and finning prohibitions;

(b) for marine turtles, seabirds, and marine mammals: fisheries bycatch mitigation measures, conservation and relevant management measures, prohibitions, and other measures in accordance with relevant international agreements to which the Party is party. (destaques nossos)

Assinala-se, finalmente, que mesmo que certos acordos internacionais de proteção ambiental - como os mencionados, ou, ainda, a Convenção Interamericana para a Proteção do Atum Tropical ${ }^{24}$ - não pudessem ser sustentáculo de obrigações no âmbito do TPP por não vincularem a integralidade dos seus membros, outros tratados transversais à tutela da biodiversidade o são.

É o caso do Protocolo de Montreal sobre Substâncias que Empobrecem a Camada de Ozônio - em vigor internacional desde 1 de janeiro de 1989, e que

23 AGENCE FRANCE-PRESSE. Japan asked to prove its whaling is for scientific research. The Guardian. 2015. Available in: <http://www.theguardian.com/environment/2015/jun/19/japanasked-to-prove-whaling-for-scientific-research>. Access: 13 abr. 2016.

24 Inter-American Tropical Tuna Convention (IATTC), da qual são signatários Canadá, Japão, México, Peru e Estados Unidos. O compromisso, destinado ao manejo sustentável de atum e outros espécimes do Pacífico-leste, foi objeto de um recente Informe do U.S. National Oceanic and Atmospheric Administration ao Congresso daquele país, em razão de violações cometidas pelo México, incluindo pesca ilegal de tubarões, descarga de dejetos no mar e morte de baleias pelo uso de técnicas de pesca inapropriadas. (NATIONAL OCEANIC AND ATMOSPHERIC ADMINISTRATION. Improving International Fisheries Management. Available in: <http://www.nmfs. noaa.gov/ia/iuu/msra_page/2015noaareptcongress.pdf>. Access: 25 abr. 2016. 
passou por cinco revisões desde então - e a Convenção Internacional para a Prevenção da Poluição por Navios (MARPOL), que entrou em vigor em 2 de outubro de 1983. Os tratados e, no caso do MARPOL, os respectivos protocolos que o modificaram, foram mencionados de forma genérica no item "6" do Anexo 20-B do TPP, cujo artigo correspondente (Artigo 20) é marcado, conforme já se assinalou, por uma redação sem dispositivos mandatórios.

É de se ressaltar, ainda, que, a exemplo do que ocorreu com o regime de proteção à propriedade intelectual, que estabeleceu standards mais elevados que os existentes no âmbito do TRIPS e das convenções salvaguardadas pela Organização Mundial da Propriedade Intelectual, não haveria quaisquer óbices em recrudescer obrigações dos Estados-membros em torno da proteção ambiental com base em um novo marco normativo.

\section{RESOLUÇão de CONTROVÉRSIAS}

Uma das características marcantes do TPP é a previsão de um mecanismo de solução de controvérsias próprio que, conquanto criticável em termos de desenho institucional pela ausência de uma estrutura permanente e especializada, catalisa a competência para a análise de quaisquer questões sob o manto do acordo.

Nesse sentido, interessante é observar, também, que, mesmo em disputas envolvendo questões ambientais, o fato de o sistema de resolução de litígios ser unificado faz com que as sanções aplicáveis sejam em matéria comercial.

Isso ocorre porque, a despeito de a fase prévia ao litígio prever um aporte próprio em matéria ambiental - Environmental Consultations, objeto do Artigo 20.20, e Ministerial Consultations, objeto do Artigo 20.22), a etapa litigiosa segue o procedimento de instauração de um painel no âmbito do regime geral estabelecido pelo Artigo 28 do TPP:

2. Notwithstanding Article 28.14 (Role of Experts), in a dispute arising under Article 20.17.2 (Conservation and Trade) a panel convened under Chapter 28 (Dispute Settlement) shall:

(a) seek technical advice or assistance, if appropriate, from an entity authorised under CITES to address the particular matter, and provide the consulting Parties with an opportunity to comment on any such technical advice or assistance received; and (b) provide due consideration to any interpretive guidance received pursuant to subparagraph (a) on the matter to the extent appropriate in light of its nature and status in making its findings and determinations under Article 28.17.4 (Initial Report).

Outro ponto importante a salientar é que, devido ao caráter eminentemente recomendatório do capítulo que regula os compromissos de implementação doméstica e de cooperação transnacional em matéria ambiental, é possível que a demanda pela solução de controvérsias nesse assunto envolva, essencialmente, as políticas nacionais que, sob o manto da proteção ao meio ambiente, obstacularizem o livre comércio ou criem impedimentos à maximização dos lucros em matéria de investimentos.

O TPP é, essencialmente, um Acordo de Livre Comércio, de forma que, consoante, se assinalou, no início deste trabalho, outras pautas transversais, tais como a regulação da propriedade intelectual e os compromissos em matéria ambiental existem na medida em que podem interferir no escopo de livre comércio e de proteção aos investimentos realizados.

Esse escopo é bem sumarizado no capítulo atinente à resolução de disputas e seu alcance:

\section{Article 28.3: Scope}

1. Except as otherwise provided in this Agreement, the dispute settlement provisions of this Chapter shall apply:

(a) with respect to the avoidance or settlement of all disputes between the Parties regarding the interpretation or application of this Agreement;

(b) wherever a Party considers that an actualor proposed measure of another Party is or would be inconsistent with the obligations of this Agreement or that another Party has otherwise failed to carry out its obligations under this Agreement; or

(c) wherever a Party considers thata benefit it could reasonably have expected to accrue to it under Chapter 2 (National Treatment and Market Access for Goods), Chapter 3 (Rules of Origin and Origin Procedures), Chapter 4 (Textiles and Apparel), Chapter 5 (Customs Administration and Trade Facilitation), Chapter 8 (Technical Barriers to Trade), Chapter 10 (Cross-Border Trade in Services) or Chapter 15 (Government Procurement) is being nullified or impaired as a result of the application of a measure of another Party that is not inconsistent with this Agreement.

A inexistência de instâncias específicas para a resolução de disputas em questões relativas ao meio ambiente traz, por um lado, a ausência de expertise das instâncias deliberativas e, por outro, o risco de que a agenda ati- 
nente à preservação dos recursos naturais seja obnubilada pelo escopo de proteção ao escopo econômico dos acordos.

Além dos exemplos apresentados em capítulo anterior, os recentes casos sob a jurisdição do Sistema de Solução de Controvérsias do Tratado Americano de Livre Comércio (mais conhecido por sua sigla em inglês, NAFTA) ilustram esse ponto em matéria de investimentos.

Primeiramente, vale lembrar que o marco normativo estabelecido pela OMC não regula questões abrangidas no escopo dos investimentos, razão pela qual o seu Sistema de Solução de Controvérsias não pode ser invocado pelas partes litigantes para dirimir um litígio. Por seu turno, assim como o TPP, o NAFTA tem um capítulo próprio versando sobre a matéria, o que autoriza que se invoque o mecanismo arbitral igualmente previsto no tratado para resolver as controvérsias supervenientes.

O primeiro e recente caso, envolvendo a TransCanada Corp. e os Estados Unidos, envolve a acusação por parte da corporação de que o governo estadunidense violou dispositivo do NAFTA ao recusar um projeto criado pela Keystone XL no âmbito da indústria petrolífera sob o argumento de proteção ambiental. O país está sendo demandado na corte doméstica federal, mas também já foi objeto de uma notificação legal no sentido de que responderá a um procedimento arbitral no âmbito do NAFTA visando ao ressarcimento de 15 bilhões de dólares. ${ }^{25}$

A segunda controvérsia, no entanto, foi recentemente resolvida e consiste em precedente elucidativo sobre a submissão do marco protetivo do meio ambiente aos compromissos em matéria de investimento. A controvérsia, envolvendo a empresa estadunidense SD Mayers Inc. e o Governo do Canadá, dizia respeito a uma regulação estabelecida pelo país como política pública para cumprir com as obrigações assumidas no âmbito da Convenção de Basileia. ${ }^{26}$

A lide ${ }^{27}$, levada ao NAFTA sob o argumento de que o governo canadense feria o princípio do tratamento

25 SCHOR, Eleonora. Obama bit with NAFTA challenge over Keystone pipeline. Available in: <http://www.politico.com/story/2016/01/ keystone-pipeline-company-files-nafta-lawsuit-217423>. Access in: 25 abr. 2016.

26 Basel Convention on the Control of Transboundary Movements of Hazardous Wastes and their Disposal (1989).

27 Para um elucidativo sumário do caso vide: <http://www.iiapp. org/media/cases_pdfs/SD_Myers_v_Canada.rev.pdf $>$. nacional, ilustrou a posição do tribunal arbitral que, ampliando o conceito de investimento previsto no próprio acordo, assinalou a impossibilidade de invocar compromissos em matéria ambiental para refutar o cumprimento das agendas em matéria de investimentos:

Canada argues that the Interim Order merely established a uniform regulatory regime under which all were treated equally. No one was permitted to export PCBs, so there was no discrimination. SDMI contends that Article 1102 was breached by a ban on the export of PCBs that was not justified by bona fide health or environmental concerns, but which had the aim and effect of protecting and promoting the market share of producers who were Canadians and who would perform the work in Canada. ${ }^{28} 29$

O exemplo dos Acordos Regionais de Comércio, no mesmo sentido do que ocorre com os Acordos Bilaterais de Investimento, serve para enfatizar, finalmente, uma reconfiguração importante nos processos de produção normativa quanto às questões abrangidas em seu escopo.

Os mecanismos de solução de controvérsias têm um papel importante em conferir dinâmica e definir os limites das obrigações adquiridas pelos Estados envolvidos nos aludidos compromissos. A participação na resolução de disputas e uma posição favorável nas negociações possuem uma influência mútua, na medida em que, por meio delas, são preenchidas omissões nos tratados e criados precedentes hermenêuticos, delimitando a legalidade para seus membros. Esse aspecto, também, consiste num elemento sistêmico relacionado ao processo de barganha entre países, tanto em nível doméstico quanto bilateral, haja vista que outras lides podem influenciar os resultados de reclamações potenciais. ${ }^{30}$

28 Livre tradução do original: O Canadá argumenta que a Ordem Interina estabeleceu meramente um regime regulatório uniforme sob o qual todos foram tratados de forma equânime. A ninguém foi permitida a exportação de PCBs, de forma que não houve discriminação. SDMI argumenta que o Artigo 1102 foi violado por um banimento na exportação de PCBs, que não pode ser justificado por preocupações relativas à saúde pública ou ao meio ambiente, pois na verdade tiveram o objetivo de proteger e promover o mercado para os produtores que eram canadenses, e que desenvolveriam seu trabalho no Canadá.

29 A integralidade do julgamento está disponível em: <http:// www.italaw.com/documents/SDPeyers-Concurringon1stAward. pdf $>$. A autora agradece à Professora Kate Miles, do Lauchterpart Institute of Cambridge University, pelas críticas levantadas sobre o caso em análise.

30 SHAFFER, Gregory. Defending Interests: public-private partnerships in WTO litigation. Washington DC: Brookings Institution Press, 2003. 
Conquanto ainda seja incerto em que medida haverá a transferência de demandas tradicionalmente levadas à $\mathrm{OMC}$ para os mecanismos estabelecidos no âmbito de acordos megarregionais como o TPP, é certo que a incorporação da pauta W'TO+ e, principalmente, a implementação de um sistema acessível a atores nãoestatais levarão a uma ascensão de sua influência no próprio conteúdo das obrigações estabelecidas pelo tratado:

The WTO (like the GATT before it) only allows member states to bring disputes, even though the overwhelming majority of cases entail state practices that arguably harm private firms. In other words, state-to-state dispute resolution is the WTO norm for trade disputes, unlike ISDS [investorstate dispute settlement] resolution under BITs and FTAs. Faced with this difference, when an alternative is possible, private firms often prefer to bring an ISDS case under a BIT or an FTA, rather than a trade case under the WTO. A public and political debate on the merits of the current ISDS system has reemerged alongside the rise of the mega-regionals; but whether this debate will result in any meaningful change in ISDS practices via TPP or TTIP is yet to be seen ${ }^{3132}$

Consequentemente, em não havendo um marco normativo definido quanto aos deveres de proteção à biodiversidade e, em contraste, existindo um sistema robusto de tutela às questões de comércio e de investimento, é natural que, também, as obrigações ambientais sejam avençadas de maneira secundária e transversal às últimas.

31 Livre tradução do original: A OMC (assim como o GATT antes dela) apenas permite que os Estados-Membros tragam disputas, mesmo que a esmagadora maioria dos casos implique práticas do Estado que indiscutivelmente prejudicam a iniciativa privada. Em outras palavras, a resolução de litígios de Estado para Estado é a regra da OMC para disputas comerciais, ao contrário da resolução ISDS [solução de controvérsias do investidor-Estado] que ocorre sob os Acordos Bilaterais de Comércio e sobre os Acordos Regionais de Comércio. Em face dessa diferença, quando a alternativa é possível, as empresas privadas, muitas vezes preferem levar um caso "ISDS" à égide de apreciação do acordo bilateral ou regional, em vez de formular uma contenda comercial no âmbito da OMC. Um debate público e político sobre os méritos do atual sistema ISDS tem ressurgido juntamente ao surgimento dos acordos megarregionais. No entanto, a medida em que esse debate resultará em alguma mudança significativa nas práticas relativas à "ISDS" via TPP ou TTIP ainda está para ser vista.

32 CLYDE, Gary; CIMINO-ISAACS, Cathleen. How will TPP and T'TIP Change the WTO System? Journal of International Economic Law, v. 18, p. 679-696, 2015. p. 682.

\section{TPP E GOVERNANÇA AMBIENTAL}

Diante das considerações expostas, os principais impactos do TPP quanto ao regime internacional existente para o Direito Ambiental podem ser sintetizados em três principais aspectos.

Primeiramente, o enfoque na discricionariedade dos Estados-partes em regulamentar os mecanismos por meio dos quais a proteção ao meio ambiente deve ser efetivada termina por flexibilizar o arcabouço institucional já existente, refletindo uma redação normativa de

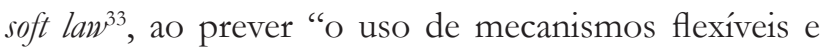
voluntários para proteger os recursos naturais e o meio ambiente em seu território", exemplificados por "auditorias e boletins voluntários, incentivos baseados no mercado, intercâmbio voluntário de informação e conhecimento especializado, e parcerias público-privadas" (Article 20.11.1) . $^{34}$

Outro aspecto que enfatiza a priorização por conservar os mecanismos institucionais e normativos domésticos em matéria de regulamentação e efetivação nas questões ambientais reflete-se nos dispositivos sobre a cooperação entre os Estados-partes. De acordo com o TPP, ela deverá ser feita "levando em consideração as prioridades nacionais e circunstâncias, e os recursos disponíveis" e "quando houver mútuo benefício dessa cooperação" (Article 20.12.2) ${ }^{35}$.

33 Para uma discussão em torno dos riscos de adoção de um sistema jurídico de tutela ao meio ambiente estruturado essencialmente em normas de soft law, ver SOUZA, Leonardo da Rocha de; LEISTER, Margareth Anne. A influência da soft law na formação do direito ambiental. Revista de Direito Internacional, Brasília, v. 2, n. 12, 2015.

34 Article 20.11: Voluntary Mechanisms to Enhance Environmental Performance 1. The Parties recognise that flexible, voluntary mechanisms, for example, voluntary auditing and reporting, marketbased incentives, voluntary sharing of information and expertise, and public-private partnerships, can contribute to the achievement and maintenance of high levels of environmental protection and complement domestic regulatory measures. The Parties also recognise that those mechanisms should be designed in a manner that maximises their environmental benefits and avoids the creation of unnecessary barriers to trade.

35 Article 20.12: Cooperation Frameworks 2. Taking account of their national priorities and circumstances, and available resources, the Parties shall cooperate to address matters of joint or common interest among the participating Parties related to the implementation of this Chapter, when there is mutual benefit from that cooperation. This cooperation may be carried out on a bilateral or plurilateral basis between Parties and, subject to consensus by the participating Parties, may include non-governmental bodies or organisations and non-Parties to this Agreement. 
Conforme se mencionou, a técnica legislativa utilizada foi caracterizada não apenas por enfatizar a dimensão voluntarista dos compromissos ambientais já pactuados pelos Estados partes, mas também por não avançar em termos prescritivos, deixando de estabelecer standards mais elevados dos que os já existentes em matéria de proteção ao meio ambiente e à biodiversidade, especificamente.

Quanto à última, organizações não governamentais como a WWF argumentam que alguns aspectos críticos, como a exemplificada pesca predatória - já que os Estados envolvidos respondem por um terço da atividade no nível mundial -, poderiam ter sido normatizadas ou articuladas com outras práticas coibidas e centrais no TPP, como as barreiras não-tarifárias e os subsídios existentes a combustíveis e os empréstimos em condições especiais à indústria pesqueira. ${ }^{36}$

Em contraponto, os Estados Unidos divulgaram em fact sheet ${ }^{37}$ sobre o TPP que propuseram e obtiveram êxito na inserção de uma provisão ${ }^{38}$ que proíbe subsídios danosos (barmful subsidies) à pesca predatória, embora a redação final do dispositivo não contenha compromissos nesse sentido:

\section{Article 20.16: Marine Capture Fisheries}

\section{$[\ldots]$ omissis $[\ldots]$}

2. In this regard, the Parties acknowledge that inadequate fisheries management, fisheries subsidies that contribute to overfishing and overcapacity, and illegal, unreported and unregulated (IUU) fishing can have significant negative impacts on trade, development and the environment and recognise the need for individual and collective action to address the problems of overfishing and unsustainable utilisation of fisheries resources.

Finalmente, é possível tecer uma análise em torno do tratado à luz da governança global. Por um lado, eventual conclusão sobre a emergência de um modelo de "governança experimentalista" (experimentalist governance) em matéria de Direito Ambiental sob o marco do TPP ainda parece precoce. O conceito foi recentemente

36 HOWARD, Brian. 4 Ways Green Groups Say Trans-Pacific Partnership Will Hurt Environment. National Geographic. Available in: <http: //news.natonalgeographic.com>. Access in: 22 maio. 2016.

37 UNITED STATES. United States Trade Representative. TPP: preserving the environment fact sheet. Available in: <www.ustr. gov/tpp>. Access in: 14 maio 2016.

38 Os dispositivos atinentes à cooperação para proteção das espécies marinhas não estavam incluídos no esboço divulgado pelo Wikileaks, em novembro de 2013. aplicado por De Búrca et al. ${ }^{39}$ ao contexto das Relações Internacionais para designar uma tendência de que, em paralelo aos regimes de governança estabelecidos pelas organizações internacionais emergentes do pós-guerra e pelas crescentes redes de cooperação complexa entre Estados e entes privados, práticas conjuntas sejam institucionalizadas e, progressivamente, revistas com base em sistemas de accountability.

Instead of the familiar combination of rules and furtive discretion they rely on what might be called 'dynamic' accountability, in which actions are justified, or compliant, if they can plausibly explained as efforts to advance organizational purpose, well informed by reflection on the best efforts of actors currently responding to like situations [...]. Experimentalism appears particularly well-suited to transnational domains, where there is no overarching sovereign with authority to set common goals even in theory, and where the diversity of local conditions and practices makes adoption and enforcement of uniform fixed rules even less feasible than in domestic settings. ${ }^{4041}$

Do ponto de vista normativo, esse sistema dá menos relevância à existência de compromissos vinculantes entre os atores envolvidos, devido à dinâmica e às especificidades técnicas que lhe são próprias, aliadas às suas peculiaridades quanto ao grau de autonomia e centralização regulatória. ${ }^{42}$

Por outro lado, ao enfatizar a soberania das partes em definir de que maneira vão reforçar o sistema jurídico internacional de proteção ambiental sem estabelecer em contrapartida mecanismos de cooperação e controles efetivos, o TPP parece não fornecer as bases para

39 DE BÚRCA, Gráinne; KEOHANE, Robert; SABEL, Charles. New Modes of Pluralist Global Governance. NYU Journal of International Law and Politics, v. 45, n. 3, p. 723-786, 2013.

40 Tradução livre do original: em vez da combinação familiar de regras e discricionariedade furtiva, baseia-se no que poderia ser chamado de accountability 'dinâmica', na qual as ações são justificadas, ou compatíveis, na medida em que possam ser plausivelmente explicadas como esforços para avançar o propósito organizacional, bem informado pela reflexão sobre os melhores esforços dos atores que atualmente respondem pelas situações [...]. Experimentalismo parece, particularmente, adaptável aos domínios transnacionais, onde não há nenhuma soberania abrangente com autoridade para definir objetivos comuns, mesmo em teoria, e onde a diversidade de condições locais e práticas torna a adoção e aplicação uniforme de regras estanques ainda menos viáveis do que em ambientes domésticos.

41 SABEL, Charles F.; ZEITLIN, Jonathan. Experimentalist governance. In: LEVI-FAUR, David (Ed.). The Oxford handbook of governance. Oxford: Oxford University Press, 2011, p. 10-14.

42 HOEKMAN, Bernard. Fostering Transatlantic Regulatory Cooperation and Gradual Multilateralization. Journal of International Economic Law, v. 18, p. 612-613, 2015. 
esse tipo de governança em termos de regulamentação:

Effective regulatory cooperation requires going beyond legally binding (i.e. enforceable) treaties between states and toward experimentalist governance. [...] [S]uch cooperation must be premised on mutual trust, which, in turn, requires mutual assessment of performance to enable regulators to assure principals (stake-holders, legislatures) that the other party can be trusted. In practice, achieving regulatory equivalence might require regulatory agencies to modern and adjust their regimes together, so that convergence occurs over time and partner countries move closer to systems that are constructed and implemented the same way. ${ }^{4344}$

No âmbito do Direito Internacional do Meio Ambiente, é importante observar que essa tendência em se adotarem estratégias de cooperação e coordenação institucional como uma alternativa às limitações do sistema internacional ambiental e dos processos de normatização que lhe são próprios vem se fortalecendo, notadamente para articular os regimes de regulação climática e biodiversidade. $^{45}$

Essa dinâmica, certamente, prevalecerá também no desenho institucional do TTIP, que, ao reunir em um mesmo acordo a União Europeia e os Estados Unidos, visa a estabelecer um compromisso conjunto para além da eliminação de tarifas - objetivo típico dos acordos de livre comércio. $\mathrm{O}$ atual esboço que, se assinado, resultará na criação da maior zona de livre comércio do mundo, conta com um inédito capítulo de cooperação internacional em matéria de regulação, o Horizontal Chapter on Regulatory Coherence (IRC), resultante da existência de um sistema de controle interinstitucional maduro no âmbito da União e do equilíbrio no poder de barganha entre

43 Livre tradução do original: Uma efetiva cooperação em matéria regulatória requer ir além de tratados legalmente vinculantes (i.e., coercitivos) entre Estados e no sentido de uma governança experimentalista. [...] Esse tipo de cooperação tem como premissa a confiança mútua, a qual, por seu turno, requer avaliação mútua da performance para permitir que os mecanismos reguladores assegurem que os órgãos de tomada de decisão (administradores, legisladores) ensejem confiabilidade nas outras partes. Na prática, conseguir uma equivalência regulatória certamente requer que agências reguladoras modernizem e ajustem seus regimes conjuntamente, de forma que esta convergência ocorra no curso do tempo e que os países parceiros caminhem em direção a sistemas que são construídos e implementados da mesma maneira.

44 HOEKMAN, Bernard. Fostering Transatlantic Regulatory Cooperation and Gradual Multilateralization. Journal of International Economic Law, v. 18, p. 612-613, 2015. p. 613.

45 ASSELT, Harro Van. Managing the fragmentation of international environmental law: forests at the intersection of the climate and biodiversity regimes. International Law and Politics, v. 44, p. 12061278, 2012. p. 1211-1212. esta e os Estados Unidos. ${ }^{46}$

Em contraste, no TPP, os impactos da ênfase na discricionariedade dos Estados-partes quanto às suas contribuições para a governança doméstica e internacional ambiental decorrem tanto da influência que outras questões amplamente disciplinadas em seu bojo podem ter sobre a agenda de proteção ao meio ambiente (investimentos, propriedade intelectual, aspectos sanitários e barreiras não alfandegárias ao comércio), quanto ao potencial que o acordo tem de se tornar um marco para futuras negociações.

Ao se considerar, ainda, o aspecto da discricionariedade, enfatiza-se uma opção normativa por descentralizar decisões atinentes à observância do marco jurídico existente, deixando-se de elevar seus padrões no nível megarregional, em consonância com o que foi feito com outros elementos da agenda WTO+, conforme se salientou no primeiro capítulo.

\section{Considerações Finais}

O marco jurídico estabelecido pelo TPP, embora ainda não possa ter quaisquer impactos auferidos, é inegavelmente relevante para compreender a dinâmica das interfaces entre comércio, investimentos e outras questões que, dada sua potencialidade de interferir nos compromissos de liberalização e livre circulação, terminaram sendo abarcadas pelo tratado.

Entre essas questões, o Direito Ambiental e a proteção à biodiversidade foram alvo de um capítulo próprio do acordo, que, em contraste com a linguagem dispensada a outros temas, é marcado pela utilização de uma terminologia vaga e recomendatória.

Conforme se assinalou, essas opções refletem, também, uma lacuna entre os acordos internacionais dos quais todos ou a maioria dos Estados signatários fazem parte e o escopo político-normativo de fazer do TPP uma instância por meio da qual tais deveres fossem reforçados.

Além disso, o artigo buscou traçar algumas provocações em torno da tendência em incorporar uma agenda

46 ALLEMANO, Alberto. The regulatory cooperation chapter of the transatlantic trade and investment partnership: institutional structures and democratic consequences. Journal of International Economic Law, v. 18, p. 625-640, 2015. 
ambiental a Acordos Bilaterais e Regionais de Comércio e a Tratados Bilaterais de Investimento, em face da possibilidade de que, uma vez entendidos como uma barreira não tarifária ou como óbice ao lucro resultante de investimentos estrangeiros, a legislação ambiental doméstica ou os tratados sobre a matéria tenham sua efetivação comprometida.

Nesse sentido, o trabalho abordou, finalmente, o papel que um sistema de solução de controvérsias que também concatene essas diversas pautas pode representar na legitimação da inobservância de deveres de preservação e sustentabilidade assumidos pelos países na seara internacional. Utilizou-se como exemplo o Sistema de Solução de Controvérsias do NAFTA e dois recentes casos que, versando sobre investimento, demandam a análise do Direito Ambiental como mecanismo para a violação do princípio do tratamento nacional.

Se é certo que muitas dessas questões remanescerão sem uma deliberação conclusiva em curto prazo, mostra-se incontroverso que o regime jurídico internacional para a tutela da biodiversidade não pode mais ser visto meramente sob o panorama do Direito Ambiental, mas sobretudo pelo papel que desempenha no escopo normativo bilateral, regional e transnacional em matéria comercial e de investimentos.

\section{REFERÊNCIAS BIBLIOGRÁFICAS}

AGENCE FRANCE-PRESSE. Japan asked to prove its whaling is for scientific research. The Guardian. 2015. Available in: <http://www.theguardian.com/environment/2015/jun/19/japan-asked-to-prove-whaling-for-scientific-research>. Access en: 13 abr. 2016.

ALLEMANO, Alberto. The regulatory cooperation chapter of the transatlantic trade and investment partnership: institutional structures and democratic consequences. Journal of International Economic Law, v. 18, p. 625-640, 2015.

ASSELT, Harro Van. Managing the fragmentation of international environmental law: forests at the intersection of the climate and biodiversity regimes. International Law and Politics, v. 44, p. 1206-1278, 2012.

CARNEIRO, Flavio L. A Parceria transpacífica: principais características e impactos sobre a regulação do comércio mundial. Boletim de Economia e Politica Internacional, n. 18, p. 60-72, set./dez. 2014.

CHILE. Dirección de Relaciones Económicas Internacionales de Chile. Minuta de las negociaciones del TPP. Disponible en: <https://www.direcon.gob.cl/wp-content/ uploads/2014/05/MINUTA_DE_LAS_NEGOCIACIONES_DEL_TPP.pdf $>$. Acceso en: 20 maio 2016.

CLYDE, Gary; CIMINO-ISAACS, Cathleen. How will TPP and TTIP Change the WTO System? Journal of International Economic Law, v. 18, p. 679-696, 2015.

DE BÚRCA, Gráinne; KEOHANE, Robert; SABEL, Charles. New Modes of Pluralist Global Governance. NYU Journal of International Law and Politics, v. 45, n. 3, p. 723-786, 2013.

GURUSWAMY, Lakshman D. The convention on biological diversity: a polemic. In: GURUSWAMY, Lakshman D; MCNEELY, Jeffrey A. (Eds.). Protection of Global Biodiversity: converging strategies. London: Duke University Press, 1998. p. 351-359.

FRIENDS FOR EARTH. The Colombia FTA: bad for the environment. Available in: <www.ffe.org>. Access en: 5 abr. 2016.

INSTITUTE OF TRANSNATIONAL LAW. NAFTA arbitration under the UNCITRAL arbitration rules. Available in: <http://www.italaw.com/documents/ SDPeyers-Concurringon1stAward.pdf $>$. Access em: 20 abr. 2016.

INTERNATIONAL COURT OF JUSTICE. Whaling in the Antartic (Australia v. Japan: New Zealand intervening). Available in: <http://www.icj-cij.org/docket/index. php?p1=3\&p2=1\&case=148>. Access: 13 abr. 2016.

NELSEN, Arthur. Japan Defies IWC Ruling on "Scientific Whaling". The Guardian. Available in: <http:// www.theguardian.com/environment/2014/sep/18/ japan-whaling-southern-ocean-iwc-ruling $>$. Access in: 15 abr. 2016.

HOEKMAN, Bernard. Fostering transatlantic regulatory cooperation and gradual multilateralization. Journal of International Economic Law, v. 18, p. 609-624, 2015.

HOWARD, Brian. 4 Ways Green Groups Say Trans-Pacific Partnership Will Hurt Environment. National Geographic. Available in: <http: //news.natonalgeographic. com>. Access in: 22 maio. 2016.

SABEL, Charles F; ZEITLIN, Jonathan. Experimentalist governance. In: LEVI-FAUR, David (Ed.). The 
Oxford handbook of governance. Oxford: Oxford University Press, 2011.

SOUZA, Leonardo da Rocha de; LEISTER, Margareth Anne. A influência da soft law na formação do direito ambiental. Revista de Direito Internacional, Brasília, v. 2. n. $12,2015$.

MELTZER, Joshua. The Trans-Pacific Partnership Agreement, the environment and climate change. In: VOON, Tania (Ed.). Trade liberalisation and international co-operation: a legal analysis of the trans-pacific partnership agreement. Camberley: Edward Elgar, 2014.

SHAFFER, Gregory. Defending interests: public-private partnerships in WTO litigation. Washington DC: Brookings Institution Press, 2003.

SCHOR, Eleonora. Obama bit with NAFTA challenge over Keystone pipeline. Politico. Available in: <http://www.politico.com/story/2016/01/keystone-pipeline-company-files-nafta-lawsuit-217423>. Access in: 25 abr. 2016.

TERRY, Simon. The environment under TPPA governan$c e$ : the law foundation New Zealand. Available in: <http://www.sustainabilitynz.org/wp-content/uploa-
ds/2016/01/TheEnvironmentUnder'TPPAGovernance_2016.pdf $>$. Access in: 20 abr. 2016.

TRANS-PACIFIC PARTNERSHIP. Acuerdo de negociación transpacifico: informe de la primera ronda de negociación. Melbourne: Australia, 2010.

UNITED STATES. National Oceanic and Atmospheric Administration. Improving International Fisheries Management. Available in: <http://www.nmfs.noaa.gov/ia/ iuu/msra_page/2015noaareptcongress.pdf $>$. Access in: 25 abr. 2016.

UNITED STATES. United States Trade Representative. TPP: preserving the environment fact sheet. Available in: <www.ustr.gov/tpp>. Access in: 14 maio 2016.

UNITED STATES. Trans-Pacific Partnership agreement. Available in: <https://ustr.gov/tpp/>. Access in: 20 maio. 2016.

VILLAREAL, M. Angeles. The U.S. Colombia free trade agreement: background and issues: congressional research services. Available in: <www.crs.gov>. Access in: 10 abr. 2016. 
Para publicar na Revista de Direito Internacional, acesse o endereço eletrônico www.rdi.uniceub.br ou www.brazilianjournal.org.

Observe as normas de publicação, para facilitar e agilizar o trabalho de edição. 